\title{
Ocular complications of bungee jumping
}

This article was published in the following Dove Press journal:

Clinical Ophthalmology

3 October 2012

Number of times this article has been viewed

H Mohammed J Hassan ${ }^{1-3}$

Georgios Mariatos 1,2

Theocharis Papanikolaou ${ }^{4}$

Akshatha Ranganath ${ }^{1,2}$

Hala Hassan ${ }^{5}$

'Barnsley Hospital NHS Foundation Trust, Barnsley, UK; ${ }^{2}$ The Rotherham NHS Foundation Trust, Rotherham, UK; ${ }^{3}$ University of Sheffield, Sheffield, UK; ${ }^{4}$ North Middlesex University Hospital, London, UK; ${ }^{5}$ Corneal and External Disease Service, Moorefield's Eye Hospital NHS Foundation Trust, London, UK
Correspondence: H Mohammed J Hassan Barnsley Hospital NHS Foundation Trust, Ophthalmology Department, Barnsley, South Yorkshire, England, United Kingdom S75 2EP Email mohammed.hassan2@nhs.net
Aim: In this paper, we will try to highlight the importance of various investigations and their crucial role in identifying whether the defect is structural or functional.

Case history: A 24-year-old woman presented with ocular complications after bungee jumping. Subsequently, although all ophthalmic signs resolved, she complained of decreased vision in her left eye.

Conclusion: Initial ophthalmic injury was detected by optical coherence tomography scan showing a neurosensory detachment of the fovea. This was not initially detected on slit-lamp examination or fluorescein angiography. On later examination, although the optical coherence tomography scan showed no structural damage, electrodiagnostic tests showed a functional defect at the fovea.

Keywords: bungee jumping, optical coherence tomography, OCT, pattern electroretinogram, PERG, ocular complications

\section{Case report}

A 24-year-old female presented to the eye department complaining of significantly blurred vision in the left eye and bilateral subconjunctival hemorrhages immediately after a bungee jump from the top of a crane. There was associated nausea, headache, neck pain, and a feeling of tightness in the chest.

The patient had a history of epilepsy during childhood and was symptom-free since the age of 11 years. She was not on any medication. She had no past ocular trauma or surgery. On general examination, she was alert with a score of 15 on the Glasgow Coma Scale.

She was hemodynamically stable and all vital signs were normal. Neurological examination was normal. Computed tomography of the head and neck did not reveal any abnormalities.

Unaided visual acuity of the right eye was 0.00 LogMAR, and the left eye was 1.08 LogMAR, not improving with pinhole. Anterior segment examination revealed normal pupillary reactions to light and accommodation without a relative afferent pupillary defect. There were extensive bilateral subconjunctival hemorrhages and hematomas.

Fundoscopy revealed normal optic discs. There were a few round deep retinal hemorrhages in the left eye temporal to the optic disc along with a dull foveal reflex (Figure 1). The foveal reflex in the right eye was normal. Optical coherent tomography (OCT) of both maculae showed a localized left subfoveal neurosensory 


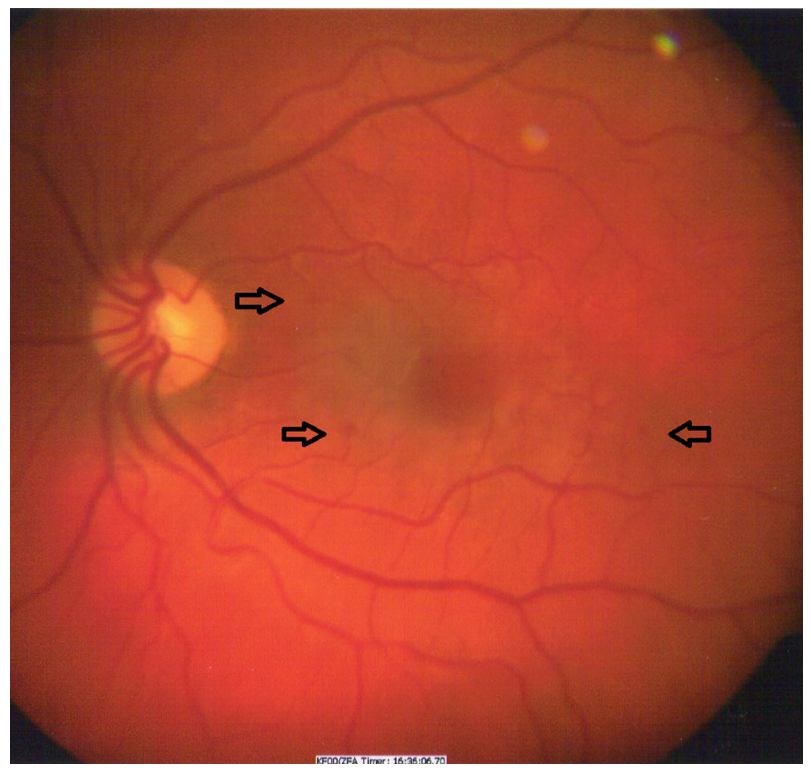

Figure I Left macula with dull foveal reflex and a few hemorrhages are indicated by arrows.

detachment (Figure 2). No abnormalities were detected in the right macula. Fundus fluorescein angiography (FFA) was undertaken, which showed the presence of hemorrhages in the left macula, without hyperfluorescence, leakage, or pooling.

A week later, the unaided vision of the right eye remained stable at 0.00 LogMAR, while the unaided vision of the left eye had improved to $0.00 \operatorname{LogMAR}$, but was subjectively blurred. Left macular hemorrhages had resolved and OCT images showed normal foveal contour with no signs of neurosensory detachment (Figure 3).

One month later, the patient was still complaining of left eye vision being blurred with no obvious corresponding clinical, biomacroscopically, or OCT imaging findings. There were no visual field defects.

Three and a half months later, subjective left eye blurred vision remained even though OCT mapping was normal in

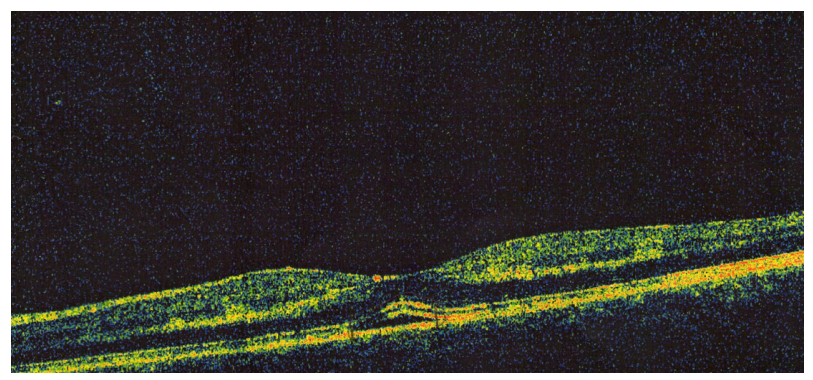

Figure 2 Localized left subfoveal neurosensory detachment.

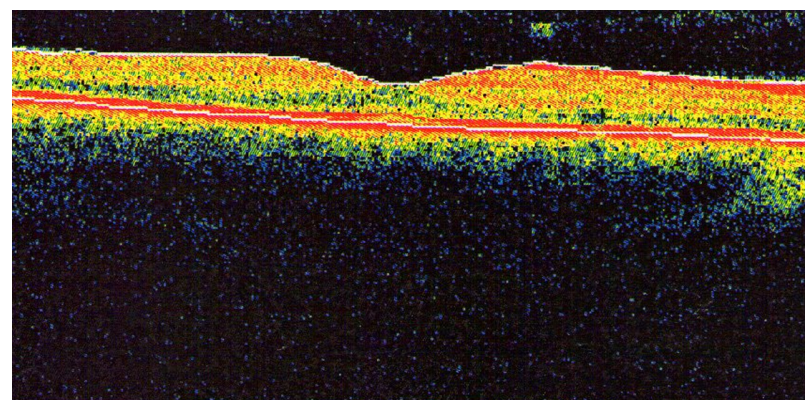

Figure 3 One week later, OCT showed normal foveal contour with no signs of neurosensory detachment.

Abbreviation: OCT, optical coherence tomography.

both eyes, and unaided vision was 0.00 LogMAR bilaterally. Electrodiagnostic tests were requested. An electroretinogram (ERG) was normal bilaterally. Pattern electroretinogram (PERG) was borderline for the right eye, and slightly subnormal for the left eye indicating a degree of macular dysfunction. Multifocal-ERG was not done because the patient felt unwell on that day.

\section{Discussion}

With the increased popularity of bungee jumping as a leisure activity over recent years, there have been reports of associated ocular complications. ${ }^{1-3}$ Most of these ocular complications were subconjunctival and benign, but they were also serious given that patients have been found with severe retinal hemorrhages. ${ }^{2-4}$ Most authors agree that the sudden increase in intrathoracic and intravenous blood pressure leads to the development of these retinal hemorrhages, ${ }^{5}$ even though the actual mechanism of this pressure increase is not clear.

Chan suggests that a sudden change in the hydrostatic pressure in the ciliary and retinal circulations that occurs in relation to the wide change of gravitational forces during the dive may be the most important etiological factor. ${ }^{4}$ This view is supported by other authors who agree that the most likely cause of these retinal hemorrhages is a sudden rise of intravascular venous pressure in the upper portion of the body during the deceleration phase of the jump in a fashion similar to Purtscher and Valsalva retinopathy., ${ }^{3,6}$ However, Innocenti and Bell suggested that breath-holding and tensing of the abdominal muscles are the main causes of the sudden rise in intrathoracic and intravenous pressure, which are thought to cause the retinal damage. ${ }^{5}$

Habib et al suggested that as the intravenous pressure increases, there is a spontaneous rupture of superficial capillaries resulting in a hemorrhagic detachment of the internal limiting membrane in the foveal region. ${ }^{7}$ 
The gravitational force (G-force) on a body is its acceleration relative to the freefall. The term G-force is technically incorrect, as it is a measure of acceleration, not force. G-forces, when multiplied by mass, are associated with a certain type of mechanical force ${ }^{8}$ Although the human body can survive high positive and negative Gs, a rapid deceleration in short duration of more than -3 Gs (negative) is enough to cause ocular injuries. ${ }^{9}$

Retinal and subconjunctival hemorrhages are the most common ocular complications, as a rapid $-3 \mathrm{G}$ deceleration will increase the intravenous pressure to $100 \mathrm{mmHg}$ or more. This is the same mechanism that produces the retinal hemorrhages in the "shaken baby syndrome." It has been calculated that the rapid \pm Gs during the shaken baby syndrome can go up to $9 \mathrm{Gs}$, resulting in hemorrhages in different anatomical layers of the retina. ${ }^{8}$ However, there are species that can handle the rapid \pm Gs without any problem, such as the woodpecker.

The woodpecker is able to use his chisel-tipped bill to hammer wood at the rate of 16 times a second, or nearly 1000 pecking blows per minute. The suddenness of this movement in which the woodpecker's head is brought to a halt during each peck results in a stress equivalent of 1000 times the G-force. His highly adapted eyes ${ }^{10}$ have an avascular retina and pectin, which play a role in maintaining an effective cushion. This pectin can be briefly filled with blood, which can elevate his intraocular pressure which, in effect, maintains a firm pressure on the crystalline lens and retina to prevent any retinal damage from a higher G-force.

Human orbits contain enough quantity of orbital soft tissue (retrobulbar fat pads), tight fibrous compartments, and ligaments allowing for a freer, but controlled, globe movement within the orbit. Without a structure containing pectin and acting as a vascular cushion (as is the case in the woodpecker's orbits), a bungee jumper (or infant in shaken baby syndrome) is subject to excessive accelerationdeceleration forces, and he or she would, obviously, develop ocular complications. Optic nerves are firmly attached to sclera and to the orbital apex, so violent translational movements of the globe can potentially generate severe traction forces at these sites.

The mechanical effect of the G-force on vitreous movement also contributes to retinal injuries by avulsing at the anterior segment of the vitreous base, causing oral retinal tears, retinal detachments, vitreous hemorrhages, posterior vitreous detachment, and posterior macular holes. Furthermore, there is another pathophysiological cause of the ocular injuries due to bungee jumping, which is the Valsalva phenomenon's effect on the eye.

The patient's normal ERG - a test of retinal photoreceptors - and a test of inner nuclear layer function showed no evidence of diffuse retinal dysfunction; however, the abnormal result of the PERG test - a test of retinal ganglion cell function - is consistent with a degree of macular dysfunction, particularly in the left eye of our patient.

The limitation of our report is that it is based on a single case. We could not comment on our patient's multifocal ERGS as she was unable to perform this test and we had to abandon the procedure.

\section{Conclusion}

Our case report highlights the importance of tests such as OCT and PERG in the diagnosis of posttraumatic macular cone dysfunction. We were able to point out a plausible cause that might be able to explain the patient's complaint of "slightly blurred central vision in the left eye." The posttraumatic macular neurosensory detachment resolved, and the visual acuities were -0.00 LogMAR, bilaterally. Indeed, the OCT showed no structural damage, but the electrodiagnostic tests showed a functional defect at the fovea.

Ultimately, bungee jumpers need to make sure that stringent safety guidelines are implemented by the bungee jump provider.

\section{Disclosure}

The authors report no conflicts of interest in this work.

\section{References}

1. Hanbury PH. Bungy jumping. Aust N Z J Ophthalmol. 1990; 18(2):229.

2. Jain BK, Talbot EM. Bungee jumping and intraocular haemorrhage. Br J Ophthalmol. 1994;78(3):236-237.

3. David DB, Mears T, Quinlan MP. Ocular complications associated with bungee jumping. Br J Ophthalmol. 1994;78(3):234-235.

4. Chan J. Ophthalmic complications after bungee jumping. $\mathrm{Br} J$ Ophthalmol. 1994;78(3):239.

5. Innocenti E, Bell TA. Ocular injury resulting from bungee-cord jumping. Eye (Lond). 1994;8(Pt 6):710-711.

6. Van Rens E. Traumatic ocular haemorrhage related to bungee jumping. Br J Ophthalmol. 1994;78(12):948.

7. Habib NE, Malik TY. Visual loss from bungee jumping. Lancet. 1994; 343(8895):487.

8. g-force [webpage on the Internet]. San Francisco: Wikimedia Foundation, Inc. Available from: http://en.wikipedia.org/wiki/G-force. Accessed October 14, 2011.

9. Byrnes VA. Elevated intravascular pressure as an etiologic mechanism in the production of eye injuries. Trans Am Ophthalmol Soc. 1959;57: 473-538.

10. Wygnanski-Jaffe T, Murphy CJ, Smith C, et al. Protective ocular mechanisms in woodpeckers. Eye (Lond). 2007;21(1):83-89. 


\section{Publish your work in this journal}

Clinical Ophthalmology is an international, peer-reviewed journal covering all subspecialties within ophthalmology. Key topics include: Optometry; Visual science; Pharmacology and drug therapy in eye diseases; Basic Sciences; Primary and Secondary eye care; Patient Safety and Quality of Care Improvements. This journal is indexed on
PubMed Central and CAS, and is the official journal of The Society of Clinical Ophthalmology (SCO). The manuscript management system is completely online and includes a very quick and fair peer-review system, which is all easy to use. Visit http://www.dovepress.com/ testimonials.php to read real quotes from published authors. 